\title{
Critical strain energy release rate for rubbers: Single edge notch tension vs. pure shear tests
}

\author{
David Roucou • Julie Diani - Mathias Brieu • Armel Mbiakop-Ngassa
}

Received: date / Accepted: date

\begin{abstract}
In order to estimate mode I fracture strain energy release rate of a rubber upon monotonic loadings, the material is submitted to pure shear and single edge notch tension tests. Catastrophic failure happens suddenly for both tests, revealing mirror-like crack surfaces, assessing the fragile fracture. Nonetheless, Griffith failure analysis could be carried out on pure shear tests only. This analysis leads to an energy release rate value that allows challenging approximate expressions existing in the literature for pure shear and single edge notch tension tests. The pure shear approximate expression provides quantities that match the Griffith analysis. Meanwhile, the strain energy release rate values calculated directly from the single edge notch tension tests differ significantly from the values obtained in pure shear. This discrepancy is explored and possible explanations are discussed showing that pure shear tests should be favored.
\end{abstract}

Keywords Fracture - Rubber - Pure shear - SENT . Strain energy release rate

D. Roucou · M. Brieu

LaMcube, Ecole Centrale de Lille, Bd Paul Langevin, 59650

Villeneuve d'Ascq, France

E-mail: david.roucou@centrale.centralelille.fr

E-mail: mathias.brieu@centralelille.fr

J. Diani

LMS, Ecole Polytechnique, Route de Saclay, 91128 Palaiseau, France

E-mail: julie.diani@polytechnique.edu

A. Mbiakop-Ngassa

Manufacture Française des pneumatiques Michelin, CERL, Ladoux, 63040 Clermont-Ferrand, France

E-mail: armel.mbiakop-ngassa@michelin.com

\section{Introduction}

Rubber materials are polymers capable of very large deformations. In the presence of cracks, catastrophic fragile failure may be witnessed. In a seminal contribution, Rivlin and Thomas (1953) applied Griffith (1921) fracture analysis for rubber samples of various geometries including pure shear and single edge notch tension (SENT) tests. In order to carry out this analysis, several specimens presenting notches of different lengths were stretched while recording their elastic stress-stretch responses until break. When initial cracks are small enough, mode I fracture is witnessed revealing mirror-like crack surfaces. While the Griffith analysis was possible for pure shear tests, it was not for SENT ones, and in the latter case the authors proposed an analytical expression for the strain energy release rate based on geometrical considerations. Note that this expression has often been used (Lee and Donovan, 1985; Hamed and Park, 1999; Gherib et al., 2010; Gabrielle et al., 2011; Diani et al., 2015) even out of its range of application. For instance, when the initial notch is larger than a fifth of the specimen width (Aït Hocine et al., 1996; El Yaagoubi et al., 2017), due to the high deformability of rubbers, the specimen may rotate causing some shearing and consequently, mixed mode fracture. A recent contribution (Roucou et al., 2018) based on extensive SENT tests run on styrene butadiene rubbers, proved that the expression proposed by Rivlin and Thomas (1953) is difficult to validate experimentally. Since successful pure shear tests for rubbers were also made (De and Gent, 1996; Tsunoda et al., 2000), this test is chosen to characterize mode I critical strain energy release rate $\left(G_{I c}\right)$ of an unfilled rubber material. The Griffith analysis performed without approximation provides a reference value for $G_{I c}$. Then, SENT tests are performed 
in order to evaluate the same fracture quantity using Rivlin and Thomas (1953) expression. Finally, the comparison between both estimates of the strain energy release rate is discussed.

The paper is organized as follows. First, section 2 gives a brief reminder of the theory of cracks and catastrophic fragile fracture. Then, section 3 introduces the material and the experimental procedures. Results are presented in section 4 opening a discussion in section 5 . Final remarks conclude the paper.

\section{Theory}

In order to predict the propagation of cracks in elastic fragile materials without knowing the complex state of strain near the crack-tip, Griffith (1921) proposed an approach based on the energy balance between the change in elastic strain energy and the energy required to create crack free surfaces. Figure 1 illustrates the difference of stored elastic energy between the load and unload of a theoretical sample presenting initially a crack of length $a$, that has been loaded until reaching displacement $L$, while the displacement was maintained constant the crack reached length $a+d a$, and the sample was unloaded. This elastic energy difference balances the energy needed to witness the increase of the crack from length $a$ to length $a+d a$. Since fragile fracture is sudden and catastrophic, it is not possible to load a sample presenting an initial crack of length $a$, control the crack propagation and unload the specimen to compare its elastic responses between load and unload. Therefore, several specimen presenting various initial crack lengths are loaded until break. Then, for any stretch value $\lambda$ it is possible to calculate the stored elastic energy $U$, and then calculate the strain energy release rate $G$ defined as:

$G=-\left.\frac{\partial U}{\partial A}\right|_{\lambda}$

with $A$ the area of crack surfaces. The critical energy release rate $G_{c}$ corresponds to the value of $G$ when catastrophic failure occurs.

We are now considering two geometries of specimen designed to measure $G_{c}$ when the crack is submitted to mode I opening.

For the pure shear geometry (Fig. 2), four regions of different states of strain are witnessed along the sample width. Using Rivlin and Thomas (1953) notations, region $\mathrm{A}$ is load-free, region $\mathrm{B}$ is in a pure shear state, region $\mathrm{C}$ is submitted to a complex state of strain, and finally region $\mathrm{D}$ is strongly impacted by the force-free edge. Assuming a crack growing of length $d a$, the area of region $\mathrm{C}$ remains unchanged but translates of $d a$, and

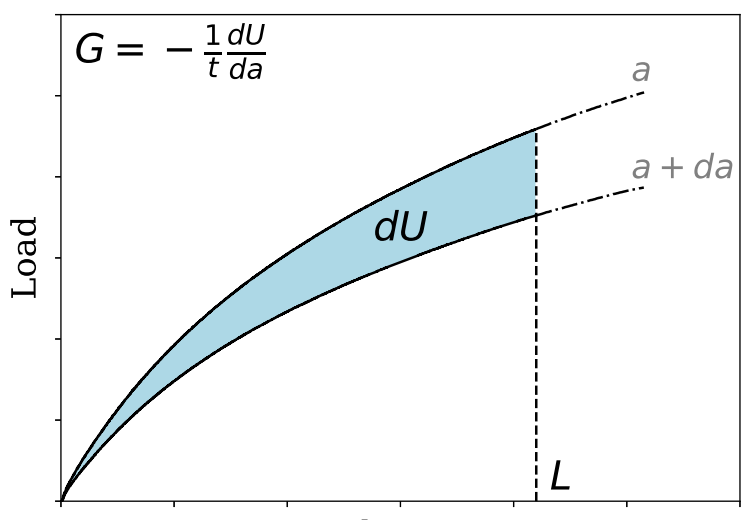

Displacement

Fig. 1 Griffith energy balance theory.

since region D remains unchanged, the pure shear region $\mathrm{B}$ decreases and the region A area increases. Therefore, during the growth of the crack, a volume of material, $V=t \times h_{0} \times d a$ with $t$ and $h_{0}$ the sample thickness and height, that was in pure shear becomes load-free, while elastic strain energies stored in regions $C$ and $D$ remain unchanged. From this statement, Rivlin and Thomas (1953) were able to calculate the strain energy release rate as,

$G(\lambda)=W_{P S}(\lambda) h_{0}$

with $W_{P S}$ the material elastic stored energy density when submitted to pure shear.

In theory, mode I crack opening may be studied also with single edge notch tension tests (Fig. 3). When the notch is smaller than one fifth of the specimen width $w$, rubber specimens of such geometries display a rather homogeneous uniaxial tension state of strain except near the crack tip, causing mode I crack opening. Nonetheless, as long as notches remain smaller than this length the recorded stress-stretch responses of SENT specimens coincide with the stress-stretch response of unnotched samples, rendering impossible to carry out a classic Griffith analysis (Rivlin and Thomas, 1953; Greensmith, 1963; Roucou et al., 2018). Therefore, Rivlin and Thomas (1953) proposed an analytical expression to calculate $G$, based on an extension of the classic infinitesimal strain analysis combined with some experimental results, that writes as:

$G(a, \lambda)=2 a K(\lambda) W_{U T}(\lambda)$

with $W_{U T}$ the material elastic stored energy density when submitted to uniaxial tension. Based on experimental work (Greensmith, 1963) and numerical analysis (Lindley, 1972), the factor $K$ has been defined as,

$K(\lambda) \simeq \frac{3}{\sqrt{\lambda}}$ 
Table 1 Material mass composition before vulcanization.

\begin{tabular}{ll}
\hline Ingredient & SBR0 \\
\hline SBR & 100 \\
Sulfur & 2.3 \\
6PPD & 1.9 \\
Steraic acid & 2 \\
ZNO & 2.5 \\
CBS & 2.3 \\
\hline
\end{tabular}

From an experimental point of view, the general nature of expression $K$ was never validated beyond Greensmith's work that dealt with unfilled natural rubbers. From a numerical point of view, several studies (Yeoh, 2002; Timbrell et al., 2003; Legrain et al., 2005) have shown that this expression is dependent of the plane stress state, which is not always easy to ensure experimentally, and of the strain energy density characterizing the rubber material.

\section{Materials and Experiments}

\subsection{Materials}

An unfilled styrene butadiene rubber (SBR) gum prepared by Michelin is considered for this study. This material is a star-branched solution presenting a molar mass of $M_{n}=120 \mathrm{~kg} / \mathrm{mol}$ which mass composition is listed in Table 1. The styrene content is $15 \%$, and the entanglement density is close to $42 \cdot 10^{-5} \mathrm{~mol} / \mathrm{cm}^{3}$. This rubber gum was chosen for presenting low viscosity, no crystallization and no Mullins softening. This rubber's mechanical behavior is close to hyperelasticity when stretched at low strain rates. Some viscoelastic dissipation may occur in the crack tip vicinity, however this should not be dominant due to the confined area involved. Therefore, it is reasonable to assume that the major source of energy dissipation stands in the crack propagation. Hence, before the crack propagation, the elastic stored energy may be conveniently estimated by computation of the external work.

\subsection{Pure Shear Protocol}

Rubber strips of $40 \mathrm{~mm}$ height and $146 \mathrm{~mm}$ width were cut in manufactured $2.4 \mathrm{~mm}$ thick rectangular plates of dimensions 141 x $146 \mathrm{~mm}^{2}$. Notches were handmade with a razor blade. Their approximate lengths were measured with a ruler and ranged between 20 and 70 $\mathrm{mm}$. The mechanical tests were carried out with an Instron 5882 machine using a $2 \mathrm{kN}$ load cell and pneumatic grips equipped with large rubber coated jaw faces (150 $\mathrm{mm})$. Once tight into the clamps, the sample effective height is recorded at $h_{0}=24 \mathrm{~mm}$. The possible slipping at the grips was estimated to be smaller than $1 \mathrm{~mm}$. Two unnotched and 13 notched samples were loaded up to failure at a constant crosshead speed of $5 \mathrm{~mm} / \mathrm{min}$, corresponding to a strain rate of $3.5 \cdot 10^{-3} \mathrm{~s}^{-1}$.

\subsection{SENT Protocol}

Long rectangular strips of width $w=30 \mathrm{~mm}$ were punched out of the same SBR plates. Notches were again handmade with a razor blade but their lengths $a$ were precisely measured with a microscope, since this quantity appears in Eq. (3) that will be used later to calculate $G_{c}$. Values of $a$ ranged between 0.89 and 5.32 $\mathrm{mm}$, remaining smaller than a fifth of the sample width $w$. The SENT tests were run on the same Instron machine using pneumatic grips. The sample length between the grips has been set to $80 \mathrm{~mm}$ ensuring uniaxial tensile strain. Specimens were stretched at a constant crosshead speed of $20 \mathrm{~mm} / \mathrm{min}$, corresponding to a strain rate of $4 \cdot 10^{-3} \mathrm{~s}^{-1}$ similar to the strain rate applied in pure shear. A total of two unnotched and 22 notched samples were tested.

\section{Results}

\subsection{Experimental results}

Cracks deviations in SBR compounds have been reported in literature (Gent et al., 2003; Marano et al., 2014; Roucou et al., 2018). Since the studied material is unfilled and does not crystallize, such deviations were not recorded during either pure shear or SENT tests, and cracks followed a rather straight path as it is illustrated in Figure 4 for pure shear tests.

Figure 5 presents the recorded load with respect to the applied displacement for pure shear tests. As predicted by Griffith theory, the load-displacement curves are ordered according to the cut length, the specimen with the longer cut being more compliant. For each notched sample, the load increases with the displacement, then starts to decline progressively to finally drop nearly instantly to zero. The sudden drop of loading characterizes the sudden unstable propagation of the crack. In terms of crack propagation, we observe that after an initial phase of seemingly purely elastic deformation, with no visible crack growth, the crack starts to propagate relatively slowly. The crack tip displacement rate was estimated, from video recording, to be smaller than $10 \mathrm{~mm} . \mathrm{s}^{-1}$. This slow propagation phase is followed by a quasi instantaneous break of the sample. Ad- 


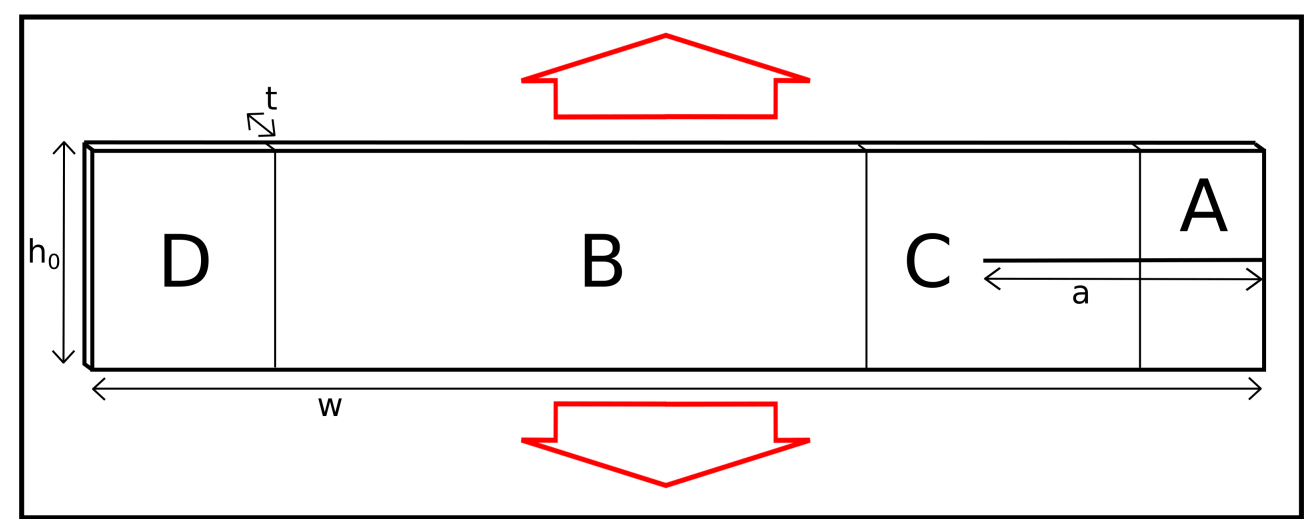

Fig. 2 Pure shear specimen with a notch length $a$ submitted to tension resulting in mode I crack opening. Regions A, B, C and $\mathrm{D}$ undergo different state of strains, region $\mathrm{B}$ being in pure shear state.

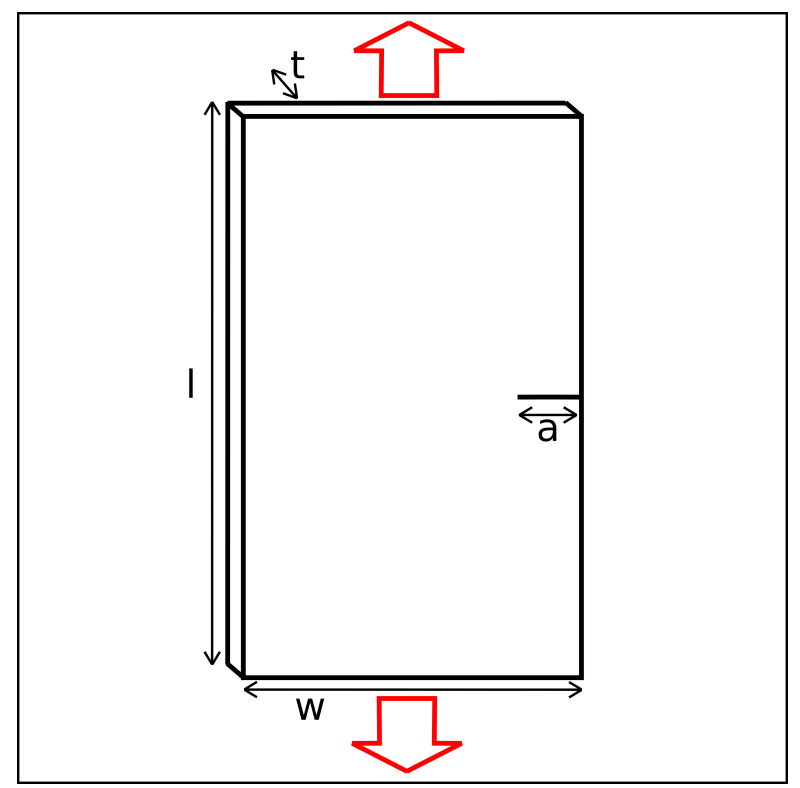

Fig. 3 SENT specimen presenting an initial notch length $a$.

ditionally, the two propagation phases can also be differentiated by observing the crack surfaces (Fig. 6). The slow propagation phase was found to leave rough crack surfaces whereas the catastrophic failure shows mirrorlike smooth surfaces proving brittle fracture. The transition point between steady tearing and catastrophic fragile failure defines a critical point in tearing mechanisms corresponding to the critical value of energy release rate $G=G_{c}$ according to Rivlin and Thomas (1953) criterion. Note in Figure 5 that the displacement at which catastrophic tearing happens, is not dependent of the initial notch length in accordance with Eq. (2).

Figure 7 presents the recorded load with respect to the applied displacement for SENT tests. Each curve superimposes with the reference load-displacement response of the unnotched sample. Specimen failure is identified by the sudden drop to zero load. These results are in good agreement with the observations reported by Rivlin and Thomas (1953) and were also observed for filled rubbers (Roucou et al., 2018). The only noticeable difference between the specimen responses stands in the displacement at break that decreases when the notch length increases. Observations of the crack surfaces indicate that, like in pure shear, steady tearing mechanisms occur at the beginning of the crack propagation revealing rough surfaces just after the handmade razor cut (Fig. 8). Nonetheless, these mechanisms are quick to let catastrophic brittle fracture to develop. Therefore, the sample fractures are similar for both geometries, and similar values of the critical energy release rate are expected for the catastrophic fragile fracture.

\subsection{Evaluation of $G$}

The superimpositions of the load-displacement of SENT specimens (Fig. 7) render impossible the Griffith analysis, while the experimental results displayed by pure shear tests in Figure 5, provide material to calculate the energy release rate $G$ according to Griffith analysis. The energy release rate may be calculated at any given stretch ratio $\lambda=h / h_{0}$, with $h$ and $h_{0}$ the current and reference sample heights, by calculating the elastic energy $U$ stored upon reaching the given stretch for every specimen characterized by their initial cut length $a$, and applying Eq. (1). Figure 9 present the values $U(a)$ calculated for several stretch ratios. For each $\lambda$, $U(a)$ is well approximated by a linear function (Fig. 9), which slope gives access to a value of $G(\lambda)$ plotted in Figure 10 with red dots. The error bars are determined with the standard deviations resulting from the linear regressions. The strain energy release rate $G(\lambda)$ is also estimated using Rivlin and Thomas analysis resulting in Eq. (2). Values of $W_{P S}$ were calculated thanks to the 


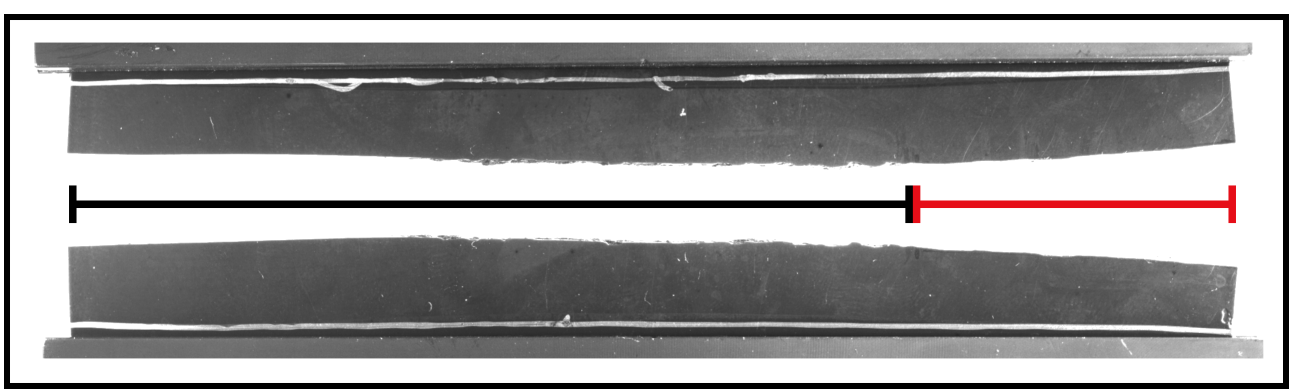

Fig. 4 Straight crack propagation recorded during a pure shear test. The initial length of the handmade razor notch is indicated in red and the straight propagation in black.

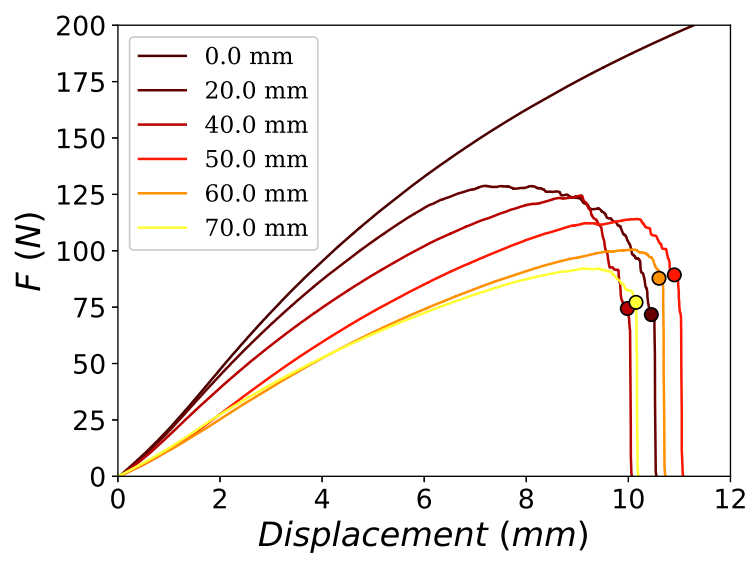

Fig. 5 Load versus displacement recorded during pure shear tests. Legend indicates the initial length of the notch for each sample. Symbols indicate critical propagation onset.

unnotched sample load-displacement response. In order to account for possible material slippage at the grips, estimated at $1 \mathrm{~mm}$, Eq. (2) was determined for initial length $h_{0}$ and for $h_{0}+1 \mathrm{~mm}$, providing us with an estimate of the error drawn by experiments. A satisfactory comparison between both evaluations of $G(\lambda)$ is shown in Figure 10, validating the convenient use of Eq. (2) to estimate the strain energy release rate $G$.

In the next section we evaluate the critical energy release rate $G_{c}$ corresponding to the value of $G$ for which catastrophic break happens.

\subsection{Critical Energy release rate $G_{c}$}

The critical stretch ratio, $\lambda_{c}$, at which the load suddenly drops to zero, is easily accessible for each test, and values of $G_{c}=G\left(\lambda_{c}\right)$ are estimated in pure shear applying Eq. (2). Figure 11 shows a rather narrow scatter of values, with an average at $3.36 \mathrm{~mJ} / \mathrm{mm}^{2}$. This value characterizing pure shear mode I fracture in the
Table 2 Critical energy release rate values given by Rivlin and Thomas expressions.

\begin{tabular}{lll}
\hline $\mathrm{Gc}\left(\mathrm{mJ} / \mathrm{mm}^{2}\right)$ & Mean value & Standard deviation \\
\hline SENT & 1.29 & 0.22 \\
PS & 3.36 & 0.20 \\
\hline
\end{tabular}

unfilled rubber, may be seen as a reference value supported by the previous Griffith analysis (Fig. 10).

As mentioned earlier, the Griffith analysis could not be carried out on SENT experimental data. Therefore, Rivlin and Thomas analysis, estimating $G$ with Eq. 3 is applied to analyze SENT tests. Values of $G_{c}=G\left(\lambda_{c}\right)$ are calculated and presented in Figure 12. While the scatter of values is again narrow, its average is approximately three times lower from the average value determined in pure shear. Discrepant results are summarized in Table 2 and possible explanations are now discussed.

\section{Discussion}

Several aspects in the testing and the experimental analysis may be source of the discrepancy. First, while similar stretch ratios were chosen, it may not draw to the same viscoelastic contributions due to the different state of strains. In the bulk, at the considered strain rates, the behavior of the rubber shows very little viscoelasticity and is close to hyperelasticity. As mentioned earlier, there may be some viscoelastic dissipation near the crack tip due to amplified local strain. However, this should not explain the large discrepancy highlighted in Table 2. Second, the major concern with critical energy release rate values obtained with SENT tests stands in the fact that it cannot be validated by a Griffith analysis. Expression Eq. (3) has not been tested extensively from an experimental point of view and therefore could be questioned. Nonetheless, the numerical analyses existing in the literature show that while it might not be 


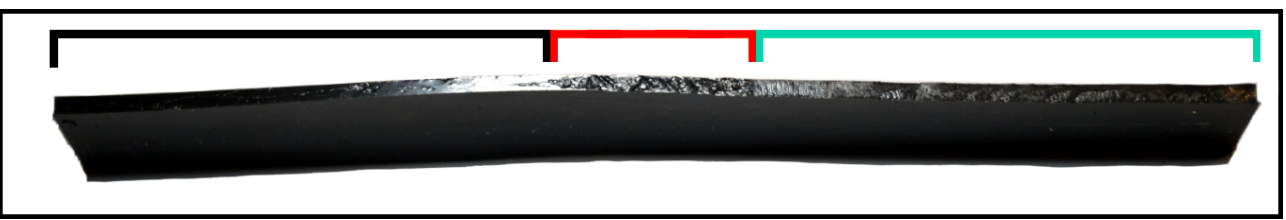

Fig. 6 Illustration of a pure shear specimen crack surface. The initial notch length appears in cyan, the rough surface due to steady crack propagation is marked in red and mirror-like smooth surface appears in black.

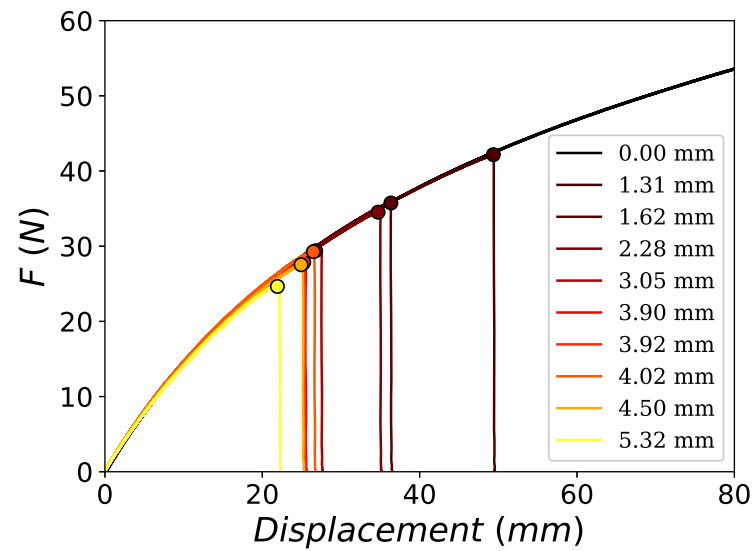

Fig. 7 Load with respect to displacement curves for SENT specimens. Legend indicates initial notch lengths. Symbols indicate critical propagation onset.

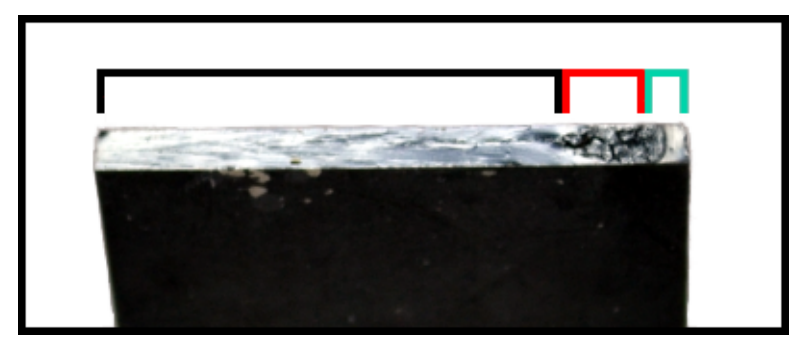

Fig. 8 Illustration of a SENT specimen crack surface. The initial notch length is marked in cyan, the rough surfaces in red and mirror-like smooth surfaces in black.

completely accurate it should not drive to such discrepancy for $G_{c}$.

The expression proposed for SENT, Eq. (3), has been applied using $\lambda_{c}$ the final stretch recorded and $a_{0}$ the initial cut length so that $G_{c}=G\left(\lambda_{c}, a_{0}\right)$. The stress strain curves show no indication of crack propagation preceding critical failure, and videos of the tests do not allow discerning elastic deformation from crack growth. However, PS tests showed that critical propagation happened after the occurrence of slow tearing yielding rough surfaces. SENT crack surfaces also revealed a small area of rough surfaces preceding the smooth surface, as shown in Figure 8. By analogy, the actual cut length at critical propagation may be differ-

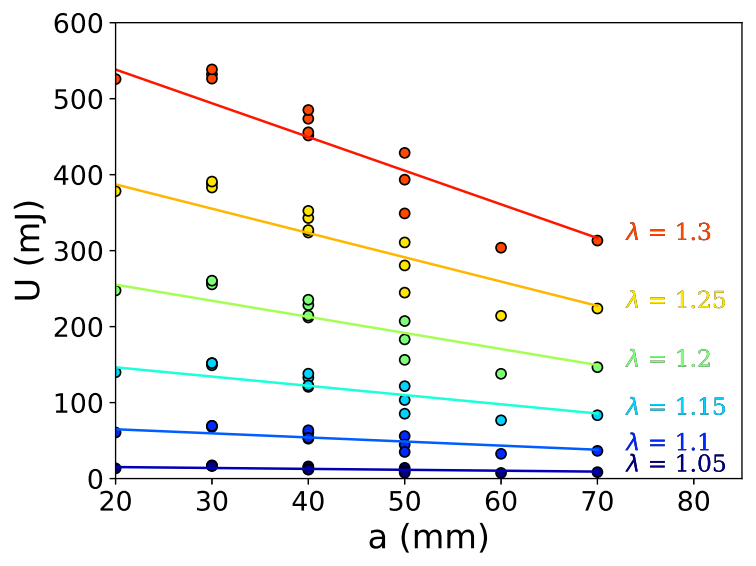

Fig. 9 Strain energy with respect to cut length calculated with data (Fig. 5) of pure shear notched specimens for several values of stretch $\lambda=h / h_{0}$.

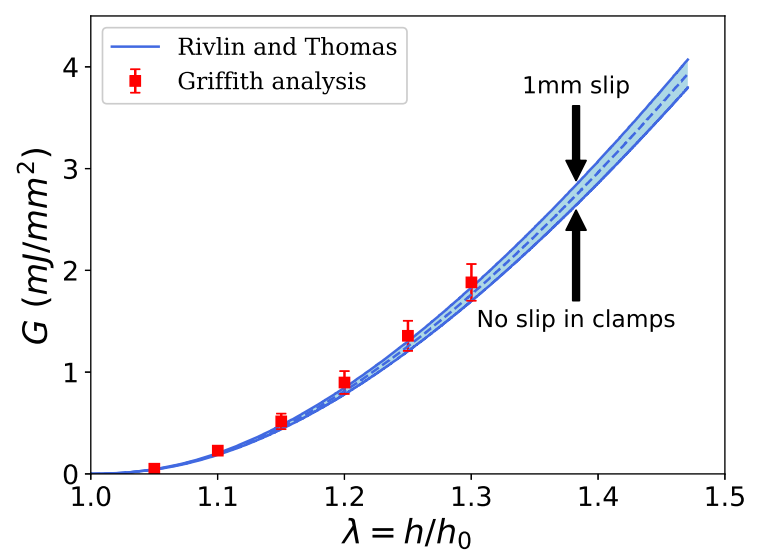

Fig. 10 Comparison of strain energy release rate values versus cut length, given by Rivlin and Thomas and Griffith methods.

ent from the initial handmade notch, due to a phase of slow crack growth highlighted by the rough surfaces. This proposition is explored by taking into account modified crack lengths for some of the SENT tests, measuring the length of the rough surface on postmortem specimen (Note that for pure shear tests, $G_{c}$ is independent of $a$ and therefore the slow propagation of the crack prior to critical failure has no impact on the cal- 


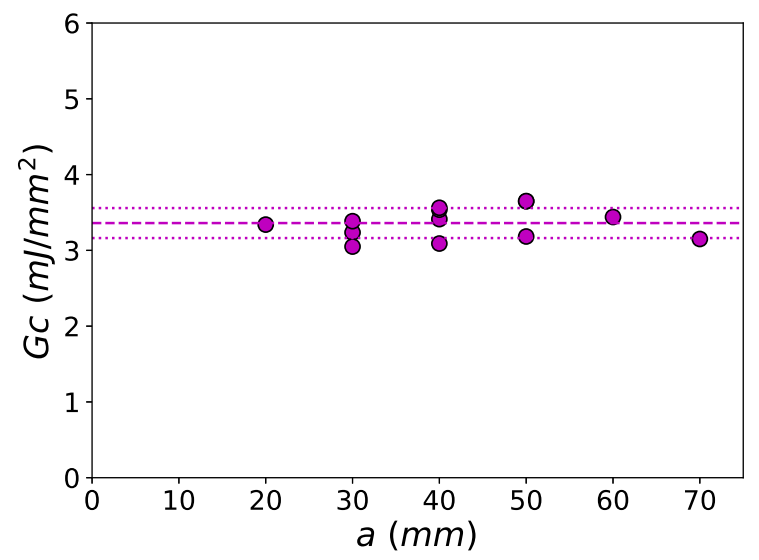

Fig. 11 Critical strain energy release rate obtained from pure shear experiments.

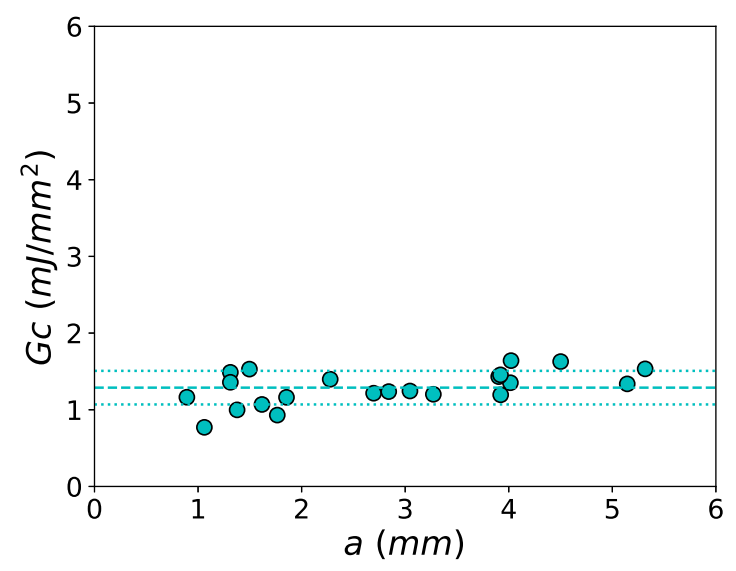

Fig. 12 Critical strain energy release rate obtained from SENT experiments.

culation of $G_{c}$ ). The measure of $a$ cannot be made precisely since the transition from rough to smooth usually happens gradually and is not constant in the specimen thickness as shown in Figure 13. Therefore, the length of this area was bounded by the minimum and maximum values $d a_{1}$ and $d a_{2}$ as indicated in the Figure 13. The normalized values of $d a_{1} / a$ and $d a_{2} / a$ are independent of $a$, ranging between 0.76 and 1.86 with an average of 1.09 for $d a_{1} / a$, and ranging between 1.49 and 3.09 with an average of 2.13 for $d a_{2} / a$.

Next, the critical energy release rates previously calculated are corrected by taking into account the total length of handmade notch plus rough surfaces for the crack length : $G_{c}=G\left(\lambda_{c}, a_{0}+d a_{i}\right)$ with $i=1$ or 2 . The values calculated for $a=a_{0}+d a_{1}$ and $a=a_{0}+d a_{2}$ should respectively be a lower and upper bound for the critical energy release rate. Values and corresponding averages are presented in Figure 14.

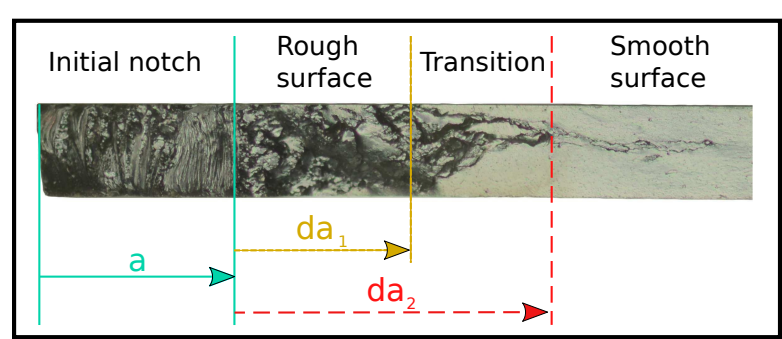

Fig. 13 Estimate of the actual crack length before catastrophic failure, as the length of the area with non smooth surface, on a post-mortem SENT specimen.

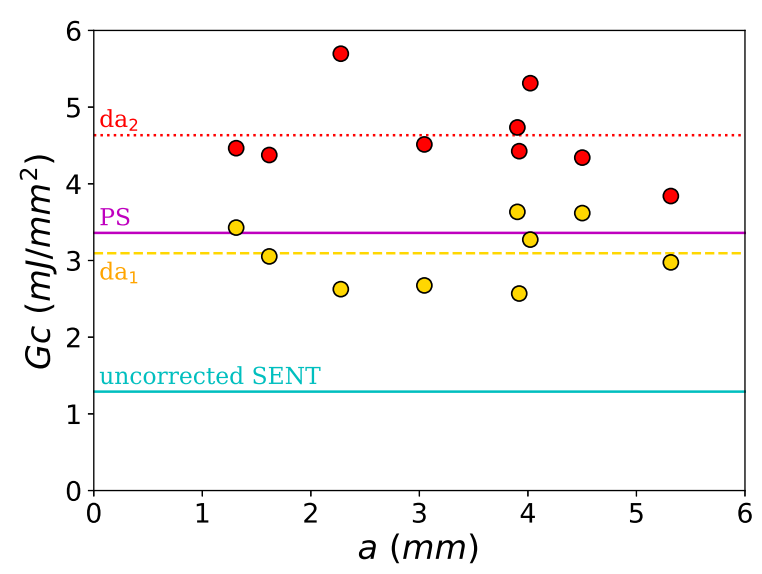

Fig. 14 Critical energy release rate values (symbols) and respective averages (broken lines) obtained with Eq. (3) for crack lengths $a_{0}+d a_{1}$ in yellow and $a_{0}+d a_{2}$ in red. The average values of $G_{c}$ obtained in pure shear (Fig. 11) and for SENT with crack lengths $a_{0}$ (Fig. 12) are also reported.

When correcting the crack lengths, values of $G_{c}$ are in good agreement with the values obtained in pure shear (Fig. 11). Note that the lengths of the cracks before the castastrophic failure may exceed one fifth of the specimen initial width. Nonetheless, this requirement is designed to limit specimen rotation and ensure uniaxial tension state of strain. The superimposition of the Force vs. displacement curves showed in Fig. 7, is an experimental validation of the uniaxial tension state of strain. Indeed, in a recent contribution (Roucou et al., 2018), it was shown that long initial crack length would introduce sample rotation characterized by Force vs. displacement curves depending on the initial crack lengths. Therefore, despite crack length exceeding one fifth of specimen width before catastrophic failure, the uniaxial state of strain seems to remain, which allows to apply Rivlin and Thomas equation (Eq. (3)). Therefore, an erroneous estimate of the crack length before break, by considering the initial notch length, could well explain the discrepancy between the original values of critical strain energy release rate found for SENT and 
PS tests, eventually validating the SENT test in turn. Note that the precise length of the crack at failure initiation is difficult to reach, preventing an accurate estimate of $G_{c}$ as shown by the scatter in Figure 14 .

In any case, the pure shear test presents several arguments in its favor,

- The experimental data give access to a genuine Griffith analysis

- The simple expression of $G$ provided by Rivlin and Thomas gives similar results as the tedious Griffith analysis making the experimental data analysis simpler

- The critical failure is independent of the initial notch length avoiding the need for its measure and a source of experimental inaccuracy.

\section{Conclusion}

In order to explore the determination of the critical energy release rate of rubbers upon Mode I fracture, an unfilled SBR gum presenting no crystallization, very low viscoelasticity and no Mullins effect, was submitted to pure shear and single edge notch tension fracture tests. A genuine Griffith analysis was successfully applied to the pure shear tests results in order to determine $G$, while the raw experimental data obtained with SENT tests would not allow it.

Using Rivlin and Thomas analysis, values of Mode I critical energy release rate were calculated for both tests with expressions Eqs. (2) and (3), revealing a severe discrepancy between these tests. In the case of the SENT tests, the critical energy release rate is assumed to be linearly dependent on the crack length before the catastrophic break. Considering the initial notch length as the crack length before catastrophic break results in underestimating the critical energy release rate significantly. Actually, catastrophic failure was preceded by some slow tearing, increasing quite significantly the crack length. Nonetheless, an accurate measure of this value seems difficult to reach rendering the use of SENT tests undoubtedly more tedious than pure shear tests. Overall, the pure shear geometry seems to present a number of advantages over SENT for experimental characterization purposes.

\section{References}

Aït Hocine N, Naït Abdelaziz M, Ghfiri H, Mesmacque G (1996) Evaluation of the energy parameter $\mathrm{J}$ on rubber-like materials: Comparison between experimental and numerical results. Engineering Fracture Mechanics 55(6):919-933.
De D, Gent AN (1996) Tear Strength of Carbon-BlackFilled Compounds. Rubber Chemistry and Technology 69(5):834-850.

Diani J, Brieu M, Batzler K, Zerlauth P (2015) Effect of the Mullins softening on mode I fracture of carbonblack filled rubbers. International Journal of Fracture 194(1):11-18.

El Yaagoubi M, Juhre D, Meier J, Alshuth T, Giese U (2017) Prediction of energy release rate in crack opening mode (mode I) for filled and unfilled elastomers using the Ogden model. Engineering Fracture Mechanics 182:74-85.

Gabrielle B, Guy L, Albouy PA, Vanel L, Long DR, Sotta P. (2011) Effect of Tear Rotation on Ultimate Strength in Reinforced Natural Rubber. Macromolecules 44(17):7006-7015.

Gent AN, Razzaghi-Kashani M, Hamed GR (2003) Why Do Cracks Turn Sideways? Rubber Chemistry and Technology 76(1):122-131.

Gherib S, Chazeau L, Pelletier JM, Satha H (2010) Influence of the filler type on the rupture behavior of filled elastomers. Journal of Applied Polymer Science 118(1):435-445.

Greensmith HW (1963) Rupture of rubber. X. The change in stored energy on making a small cut in a test piece held in simple extension. Journal of Applied Polymer Science 7(3):993-1002.

Griffith AA (1921) VI. The phenomena of rupture and flow in solids. Phil. Trans. R. Soc. Lond. A 221:163198.

Hamed GR, Park BH (1999) The Mechanism of Carbon Black Reinforcement of SBR and NR Vulcanizates. Rubber Chemistry and Technology 72(5):946-959.

Lee DJ, Donovan JA (1985) Critical J-integral and tearing energies for fracture of reinforced natural rubber. Theoretical and Applied Fracture Mechanics 4(2):137-147.

Legrain G, Moës N, Verron E (2005) Stress analysis around crack tips in finite strain problems using the eXtended finite element method. International Journal for Numerical Methods in Engineering 63(2):290314.

Lindley PB (1972) Energy for crack growth in model rubber components. Journal of Strain Analysis $7(2): 132-140$.

Marano C, Boggio M, Cazzoni E, Rink M (2014) Fracture phenomenology and toughness of filled natural rubber compounds via the pure shear test specimen. Rubber Chemistry and Technology 87(3):501-515.

Rivlin RS, Thomas AG (1953) Rupture of rubber. I. Characteristic energy for tearing. Journal of Polymer Science 10(3):291-318. 
Roucou D, Diani J, Brieu M, Witz JF, Mbiakop-Ngassa A (2018) Experimental investigation of elastomer mode I fracture: an attempt to estimate the critical strain energy release rate using SENT tests. International Journal of Fracture 209:163-170.

Timbrell C, Wiehahn M, Cook G, Muhr AH (2003) Simulation of crack propagation in rubber. Proceedings of the Third European Conference On Constitutive Models For Rubber 11-20.

Tsunoda K, Busfield JJC, Davies CKL, Thomas AG (2000) Effect of materials variables on the tear behaviour of a non-crystallising elastomer. Journal of Materials Science 35(20):5187-5198.

Yeoh OH (2002) Relation between crack surface displacements and strain energy release rate in thin rubber sheets. Mechanics of Materials 8(34):459-474. 